\title{
CONTRIBUTION A L'ÉTUDE DE L'UTILISATION DU LAIT PAR LES JEUNES ANIMAUX
}

PAR

\author{
A. M. LEROY, G. LERY, S. ZELTER
}

Laboratoire de recherches de Zootechnie de l'Institut National Agronomique

\section{PLAN DU MEMOIRE}

Première partie : Utilisation de l'énergie du lait. Exposé de la méthode expérimentale utilisée. Mesure des ingesta et excreta. Interprétation des résultats obtenus. Application pratique de ces résultats à la détermination des quantités de lait nécessaires à de jeunes animaux dont on connait le poids et le gain de poids quotidien.

Seconde partie : Utilisation de l'azote du lait. Variabilité du coefficient de rétention et de la valeur biologique de l'azote du lait, en fonction de l'énergie empruntée aux matières azotées pour équilibrer les dépenses de l'organisme Valeurs limites de ces coefficients.

\section{PREMIÈRE PARTIE :}

\section{UTILISATION DE L'ÉNERGIE DU LAIT}

\section{EXPOSE DE LA MÉTHODE UTILISEE}

Au cours d'une série de 18 expériences, poursuivies sur des agneaux, des porcelets et un veau, nous avons eu l'occasion d'étudier les échanges nutritionnels d'énergie de nos animaux, par l'emploi combiné des deux méthodes de calorimétrie indirecte, la méthode des bilans azote - carbone et la méthode des échanges gazeux.

Pendant les essais, dont la durée était d'une semaine environ, les animaux étaient placés dans des cages métalliques, munies d'un double grillage, permettant de recueillir sans pertes notables et séparément les excréments solides et les urines. Pour l'analyse des gaz de la respiration, nous nous sommes servis 
d'un appareil à confinement, formé d'une cloche métallique mobile qui pouvait être placée à volonté sur les cages, en assurant une étanchéité parfaite au moyen d'une rainure contenant de l'eau aiguisée d'acide sulfurique. La durée de chaque expérience de confinement variait avec la taille des animaux. Elle a pu atteindre jusqu'à 2 heures, pour les agneaux. L'analyse des gaz de la cloche immédiatement au début et à la fin de chaque expérience permettait de calculer les quantités d'oxygène consommé et de gaz carbonique rejeté au cours de l'essai. A la condition de multiplier les mesures, de jour comme de nuit, aux différentes heures, et de construire pour chaque semaine d'expérience les courbes exprimant les variations des échanges gazeux en fonction du temps pour une période témoin moyenne allant de o à 24 heures, nous avons pu calculer les consommations journalières d'oxygène et les émissions correspondantes de gaz carbonique avec une approximation suffisante, ainsi que nous pouvions en juger par la concordance satisfaisante des mesures effectuées aux mêmes heures, à des jours différents.

On sait, en effet, que si l'on connaît la consommation d'oxygène d'un animal en un temps donné, exprimée en litres, à o $0^{\circ}$ et sous $760 \mathrm{~mm}$ de pression, d'une part, et le quotient respiratoire $\frac{\mathrm{CO}^{2}}{\mathrm{O}^{2}}$, d'autre part, la dépense d'énergie exprimée en calories, pendant le même temps est égale au volume $v$ d'oxygène ainsi mesuré multiplié par un coefficient déduit par interpolation du tableau. ci-après :

\section{TABLEAU I}

Coefficients calorifiques de l'oxygène, en fonction du quotient respiratoire $\frac{\mathrm{CO}^{2}}{\mathrm{O}^{2}}$

\begin{tabular}{|c|c|c|c|c|c|c|c|}
\hline Valeurs de $\frac{\mathrm{CO}_{2}}{\mathrm{O}_{2}} \ldots \ldots \ldots \ldots \ldots \ldots$ & 0,70 & 0,75 & 0,80 & 0,85 & 0,90 & 0,95 & $1, \infty \circ$ \\
\hline $\begin{array}{l}\text { Calories dépensées par litre d'oxygène } \\
\text { consommé } \ldots \ldots \ldots \ldots \ldots \ldots \ldots \ldots \ldots\end{array}$ & 4,68 & 4,74 & 4,80 & 4,86 & 4,92 & 4,98 & 5,05 \\
\hline
\end{tabular}

La figure I ci-jointe, donnée à titre d'exemple, correspond au cas d'un agneau régulièrement alimenté toutes les six heures, observé pendant trois fois 24 heures. La courbe en traits pleins correspond aux dépenses exprimées en calories par heure, calculées d'après la consommation d'oxygène mesurée au cours de chaque essai. La courbe en pointillé montre la variation du coefficient respiratoire $\frac{\mathrm{CO}^{2}}{\mathrm{O}^{2}}$. Ennfin, les flèches correspondent aux heures de début des repas, et les nombres inscrits au1-dessous de chacune d'elles indiquent la quantité de lait absorbée par repas, exprimée en grammes.

D'après ces résultats, 1a dépense d'énergie de l'animal, s'est élevée à 697 calories par 24 heures. 


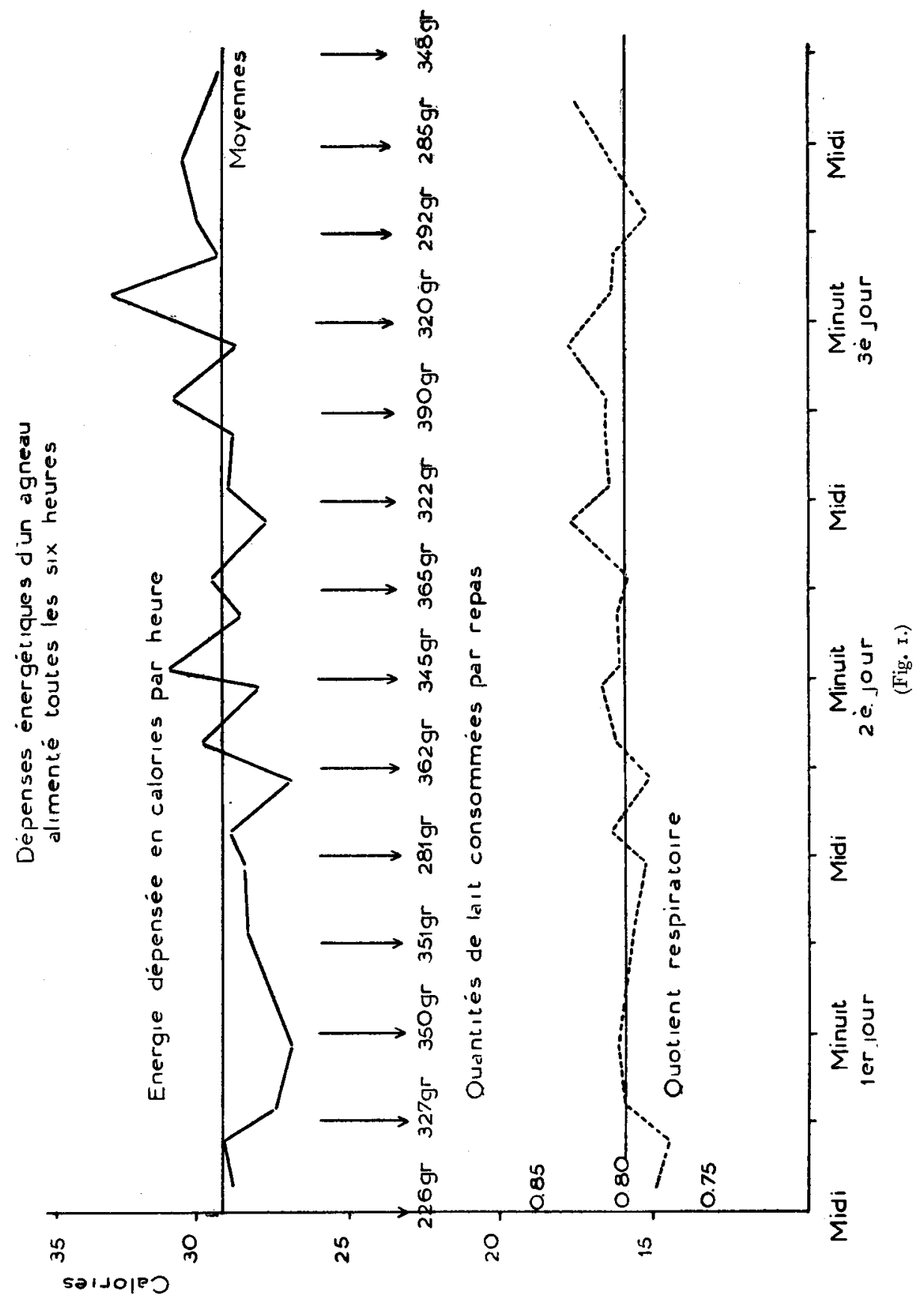


Tous les résultats figurant à la fin du présent travail ont été obtenus de cette manière.

\section{Mesure des Ingesta}

Les agneaux ont été nourris par leurs mères. Pour la détermination aussi précise que possible de leurs ingesta, nous avons utilisé le dispositif expérimental ci-après :

Les agneaux ont été pesés avant et après chaque repas, en prenant le soin d'éviter entre les pesées la défécation ou l'émission d'urine, opération relativement facile à condition de surveiller attentivement les sujets.

Afin d'établir pour chaque repas et avec le maximum d'exactitude la composition du lait ingéré, nous avons prélevé des échantillons avant et après chaque traite, sur chaque quartier de mamelle, et mélangé ces derniers à volumes égaux avant de les analyser. Pour justifier cette façon d'opérer, nous avons procédé, plusieurs fois de suite, à la traite continue de trois brebis en opérant quartier par quartier, et nous avons analysé séparément les échantillons récoltés aux différents moments de la traite. Voici les résultats de ces déterminations :

\section{TABLEAU II}

\section{Résultats de l'analyse séparée du lait extrait au cours d'une} traite, fractionnée en quatre parties égales

\section{Io Détermination du taux de matière grasse:}

Chaque détermination correspond aux moyennes de quatre opérations successives, au cours d'une période de 48 heures. Elle indique la quantité de matière grasse par kg de lait.

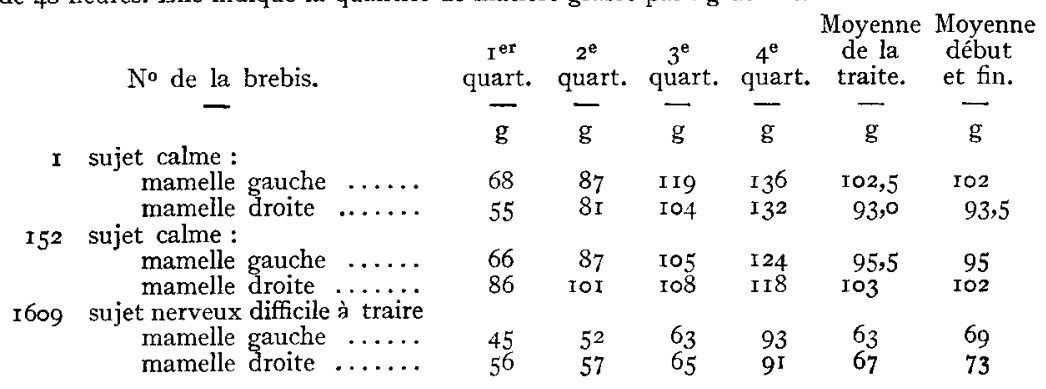

$2^{\circ}$ Détermination du taux de matière azotée :

\begin{tabular}{|c|c|c|c|c|c|c|c|}
\hline & No de la brebis. & $\begin{array}{c}\mathrm{I}^{\mathrm{er}} \\
\text { quart. }\end{array}$ & $\begin{array}{c}2^{\mathrm{e}} \\
\text { quart. }\end{array}$ & $\begin{array}{c}3^{\mathrm{e}} \\
\text { quart. }\end{array}$ & $\begin{array}{c}4^{\mathrm{e}} \\
\text { quart. }\end{array}$ & $\begin{array}{c}\text { Moyenne } \\
\text { de la } \\
\text { traite. }\end{array}$ & $\begin{array}{l}\text { Moyenne } \\
\text { début } \\
\text { et fin. }\end{array}$ \\
\hline I & mamelle gauche $\ldots \ldots$ & $\begin{array}{l}g \\
62\end{array}$ & $\begin{array}{l}\mathrm{g} \\
59\end{array}$ & $\begin{array}{r}g \\
5^{6}\end{array}$ & $\begin{array}{l}\mathrm{g} \\
55\end{array}$ & $\underset{5}{g}$ & $\begin{array}{c}g \\
5 \\
58,5\end{array}$ \\
\hline & mamelle droite $\ldots \ldots$ & 63 & $6 \mathrm{I}$ & 59 & 55 & 59,5 & 59,0 \\
\hline I 52 & $\begin{array}{l}\text { mamelle gauche } \ldots \ldots \\
\text { mamelle droite } \ldots \ldots\end{array}$ & $\begin{array}{l}67 \\
69\end{array}$ & $\begin{array}{l}64 \\
67\end{array}$ & $\begin{array}{l}59 \\
65,5\end{array}$ & $\begin{array}{l}56 \\
62\end{array}$ & $\begin{array}{l}61,5 \\
66,0\end{array}$ & $\begin{array}{l}61,5 \\
65,5\end{array}$ \\
\hline 09 & mamelle gauche.... & $5^{6}$ & 55 & 53 & $5^{2}$ & 54,0 & 54,0 \\
\hline & mamelle droite $\ldots \ldots \ldots$ & $5^{2}$ & 53 & 54 & $5^{I}$ & $5^{2,5}$ & $5^{\mathrm{I}, 5}$ \\
\hline
\end{tabular}


$3^{\circ}$ Détermination du taux d'extrait sec à $100^{\circ}$ :

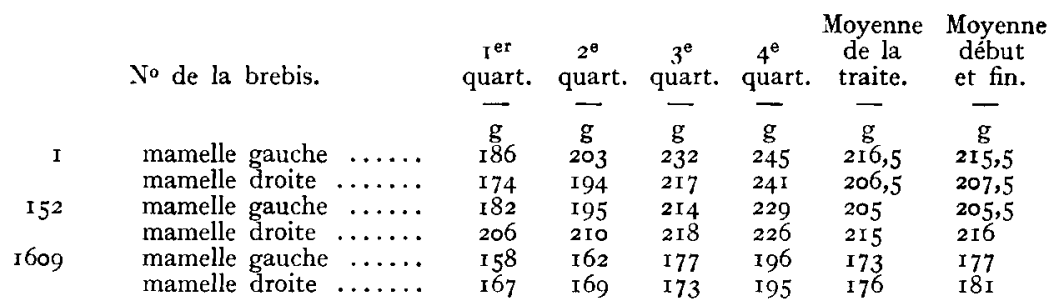

Moyennes générales

Matière grasse $. . . \ldots \ldots \ldots \ldots . .$.

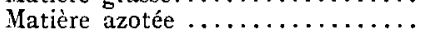

Extrait $\sec \ldots \ldots \ldots \ldots \ldots \ldots \ldots$
Moyennes entre les taux des laits de l'ensemble de la traite.

$$
\begin{array}{r}
87,3 \mathrm{~g} \\
58,6 \mathrm{~g} \\
198,7 \mathrm{~g}
\end{array}
$$

Moyennes entre les taux des mélanges de lait du début et de la fin de la traite.

$$
\begin{array}{rr}
89,2 & \mathrm{~g} \\
58,3 & \mathrm{~g} \\
200,4 & \mathrm{~g}
\end{array}
$$

La concordance entre les taux de matière grasse, de matière azotée et d'extrait sec de l'ensemble de la traite et les taux correspondants d'un mélange du début et de la fin de cette traite s'étant montrée très satisfaisante, nous avons calculé, par la suite, les ingesta avec l'aide de l'analyse d'un échantillon moyen prélevé dans les conditions précitées. L'erreur relative ainsi commise, dont 1'ordre de grandeur était au maximum de $2 \%$, pouvait être négligée sans inconvénient.

Le veau a été alimenté au seau, et les porcelets au biberon ; nous n'avons donc pas éprouvé dans ces cas particuliers de difficultés spéciales pour la mesure exacte des quantités de matériaux ingérés. Toutes ces expériences ont été effectuées dans un local à la température moyenne de $18^{\circ}$.

\section{Résultats expérimentaux}

D’après les résultats détaillés de la première expérience sur agneau, voici de quelle manière ont été effectués les calculs pour arriver à la détermination de la perte d'énergie par 24 heures, provenant du jeu des métabolismes. Nous avons ainsi combiné les deux méthodes de mesure, et pris la valeur moyenne des données fournies par chacune d'elles.

\section{Tableau III}

\section{Essais du 6 au 11 décembre}

Agneau D, âgé de 4I jours, pesant 8,9 kilogrammes.

\section{Io Mesure des ingesta par 24 heures :}

Quantité de lait. Mat. azotées. Mat. grasses. Lactose. Mat. minérales. Mat. sèche.

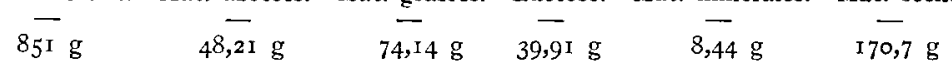


$2^{\circ}$ Mesure des excreta par 24 heures :

Fèces.

Mat. sèche. Mat. Organique.

9, $\overline{63} \mathrm{~g} \quad \overline{6,58} \mathrm{~g}$
Urine.
Quantité. Mat. Sèche. Mat. organique.

403 g $\quad$ I3,3 $\mathrm{g} \quad$ II,30 g

\section{$3^{\circ}$ Balance de l'Azote :}

Azote des ingesta. Azote fécal. Azote urinaire. Azote du croît, retenu dans l'organisme.
$\overline{7,7} \mathrm{I}$
$\overline{0,3 \mathrm{I} g}$
$\overline{3,3^{8}} \mathrm{~g}$
4,02 $\mathrm{g}$

\section{$4^{\circ}$ Balance du Carbone :}

Carbone retenu dans

Carbone des ingesta. Carbone fécal. Carbone urinaire Carbone du gaz carbonique. l'organisme.
$9 \overline{8,2} \mathrm{~g}$
$5,3 \mathrm{~g}$
$\overline{\mathrm{r}, \overline{7} \mathrm{~g}}$
$60,2 \mathrm{~g}$
$3 \mathbf{g}$

\section{$5^{\circ}$ Balance de l'Energie :}

Energie brute Energie des Energie de Energie perdue mesurable Energie retenue sous forme des ingesta. fèces. l'urine. par calorimétrie.

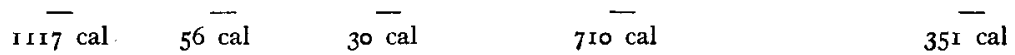

$6^{\circ}$ Détermination des éléments $d u$ crô̂t :

a) Matières azotées retenues

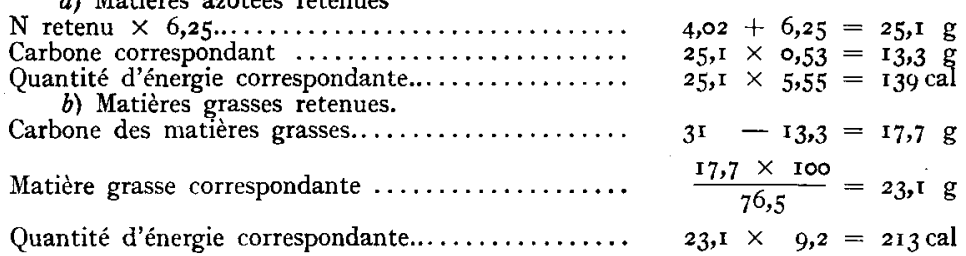

Le lecteur trouvera à la fin de cet article les données les plus caractéristiques, correspondant à chacune des I8 expériences que nous avons effectuées.

\section{Interprétation des résultats obtenus}

En groupant nos résultats d'après le tableau 4 ci-après, nous avons partagé dans chaque cas la quantité d'énergie perdue par le jeu des métabolismes en deux fractions : la première correspond à la dépense de fond, ou dépense dite du métabolisme basal, qui correspond comme nous le savons, à la dépense théorique de l'animal supposé dans l'état de jeûne et au repos complet ; la seconde est une dépense supplémentaire entraînée par la consommation des aliments, sous l'influence excitante des matériaux ingérés et des produits de leur métabolisme. 
TABLEAU IV

\begin{tabular}{|c|c|c|c|c|c|c|c|c|}
\hline \multirow{3}{*}{ No } & \multirow[b]{2}{*}{$\begin{array}{l}\text { Poids } \\
\text { du } \\
\text { sujet }\end{array}$} & \multirow[b]{2}{*}{$\begin{array}{c}\text { Energie } \\
\text { métabolisable }\end{array}$} & \multicolumn{2}{|c|}{$\begin{array}{l}\text { Energie des tissus de } \\
\text { croissance. }\end{array}$} & \multicolumn{2}{|c|}{ Energie perdue } & \multirow{2}{*}{\multicolumn{2}{|c|}{$\begin{array}{c}\text { Matière } \\
\text { sèche } \\
\text { supple } \\
\text { ingé- parg de } \\
\text { rée mat. sèche }\end{array}$}} \\
\hline & & & $\frac{\text { Mat. }}{\text { azotées }}$ & $\begin{array}{l}\text { Mat. a } \\
\text { grasses }\end{array}$ & $\begin{array}{l}\text { Fraction corr. } \\
\text { au métabolisme } \\
\text { basal }\end{array}$ & $\begin{array}{l}\text { Energie } \\
\text { supplé- } \\
\text { ment }\end{array}$ & & \\
\hline & $\overline{\mathrm{kg}}$ & $\overline{\mathrm{cal}}$ & $\overrightarrow{\mathrm{cal}}$ & $\overline{\mathrm{cal}}$ & $\overline{c a l}$ & & $\bar{g}$ & \\
\hline
\end{tabular}

Expériences avec agneaux

\begin{tabular}{|c|c|c|c|}
\hline I & $\ldots$ & 8,9 & $I \circ 3 I$ \\
\hline 2 & $\cdots \cdots$ & 9,7 & $93^{6}$ \\
\hline 3 & $\ldots$ & $\mathrm{II}, 2$ & I 680 \\
\hline 4 & $\ldots$ & 7,4 & 880 \\
\hline & $\cdots$ & 8,6 & $79^{6}$ \\
\hline & $\cdots \cdots$ & 0,2 & $\begin{array}{r}050 \\
\end{array} 027$ \\
\hline 8 & $\ldots$ & $\begin{array}{l}5,2 \\
5,8\end{array}$ & 904 \\
\hline 9 & $\ldots$ & 6,6 & 909 \\
\hline 10 & $\ldots$ & 6,0 & 734 \\
\hline I I & $\ldots$ & 6,7 & $93^{8}$ \\
\hline I & $\ldots$ & 7,4 & $5^{82}$ \\
\hline$I_{3}$ & $\cdots \cdots$ & 10,3 & I 286 \\
\hline
\end{tabular}

$\begin{array}{rrr}\text { I39 } & 212 & 498 \\ \text { I05 } & \text { I } 52 & 521 \\ \text { I72 } & 674 & 565 \\ \text { I30 } & \text { I32 } & 448 \\ \text { I25 } & 37 & 490 \\ \text { I I } & \text { I I } & 474 \\ \text { I89 } & 315 & 368 \\ \text { I32 } & 222 & 392 \\ \text { I I4 } & 221 & 423 \\ \text { I } 6 & 48 & 400 \\ \text { I58 } & 224 & 424 \\ 62 & -69 & 448 \\ 271 & 257 & 540\end{array}$

\begin{tabular}{|c|c|c|}
\hline 182 & I 70,7 & 1,07 \\
\hline I 58 & I 51,5 & $I, 05$ \\
\hline 268 & 250 & $\mathbf{I}, 07$ \\
\hline 170 & I 43 & I, I9 \\
\hline I 44 & 134,5 & I.07 \\
\hline I 44 & 136 & I,, 0 \\
\hline 165 & I 66,5 & 0,99 \\
\hline 158 & 146 & 1,08 \\
\hline $15 \mathrm{I}$ & $r_{45,5}$ & $\mathrm{I}, 04$ \\
\hline I 70 & 124,0 & I,37 \\
\hline I 32 & I 52,5 & 0,87 \\
\hline 127 & IOI & 1,26 \\
\hline 218 & 232 & 1,09 \\
\hline
\end{tabular}

Expériences avec veau

\begin{tabular}{|c|c|c|c|c|c|c|c|c|c|}
\hline I4 & $\ldots \ldots$ & 43,0 & $477 \mathrm{r}$ & $8 \mathrm{I} 6$ & I 643 & I 520 & 792 & $93^{8}$ & 0,85 \\
\hline I 5 & $\ldots \ldots$ & 44,0 & 3420 & 520 & 604 & I $53^{\circ}$ & 766 & 680 & I, \\
\hline I6 & $\cdots$ & 48,6 & 4882 & I I 22 & 973 & I 710 & 1 or 7 & 1200 & \\
\hline I 7 & $\ldots$. & 53,0 & 6731 & I IO3 & 2575 & I 770 & I 283 & I $25 x$ & $\mathbf{r}$ \\
\hline
\end{tabular}

Expérience avec porcelets ( 6 porcelets observés ensemble) - données moyennes par porcelet

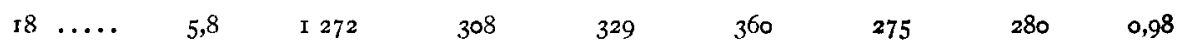

Si nous comparons dans chaque cas le supplément d'énergie provenant de cette action excitante à la quantité correspondante de matière sèche ingérée, les résultats obtenus paraissent assez sensiblement constants,surtout si l'on tient compte des erreurs expérimentales possibles. La dépense supplémentaire ainsi définie est en moyenne de $\mathrm{I}, 06$ calories par gramme de matière sèche ingérée, pour les agneaux, de $0,95 \mathrm{cal}$ pour le veau et de 0,98 cal pour les porcelets. Pour une première approximation, nous pouvons en fixer la valeur à une calorie par gramme. Cette conclusion, à la fois simple et importante, est en parfaite harmonie avec la théorie exposée à maintes reprises par l'un de nous, et qui a fait l'objet d'une communication de DELAGE et GASNIER publiée dans les comptes rendus du Ve Congrès International de Zootechnie, tenu à Paris en novembre I949.

Des conséquences pratiques utiles aux éleveurs découlent de nos observations. Il est possible, en effet, à l'aide de ce qui précède, de calculer rapidement la quantité de lait indispensable à un animal d'une espèce donnée dont on connaît à la fois le poids et le gain quotidien. Rappelons d'abord quels sont, pour les bovins, ovins et porcins, les besoins d'entretien et de production, évalués en calories d'énergie nette : 


\section{TABLEAU V}

I ${ }^{\circ}$ Besoins d'entretien, en fonction du poids vif.

\begin{tabular}{cc} 
Poids & $\begin{array}{c}\text { Veaux } \\
-\end{array}$ \\
\hline 50 & $\begin{array}{c}\text { par jour } \\
\text { p } 700\end{array}$ \\
60 & I 800 \\
70 & I 900 \\
80 & 2000 \\
90 & 2100 \\
100 & 2200 \\
110 & 2300 \\
120 & 2400
\end{tabular}

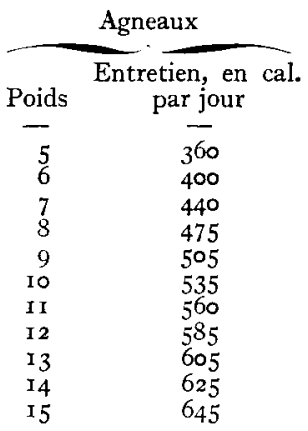

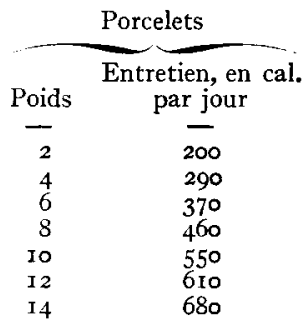

$2^{\circ}$ Besoins de production, en fonction de l'âge et de la vitesse de croissance.

Veaux :

Besoins par $\mathrm{kg}$ de gain de poids vif

\begin{tabular}{|c|c|c|c|c|c|}
\hline 11 & Gain par jour & $\begin{array}{r}500 \mathrm{~g} \\
2200 \mathrm{C}\end{array}$ & $\begin{array}{r}750 \mathrm{~g} \\
2800 \mathrm{C}\end{array}$ & $\begin{array}{l}\text { I } 000 \mathrm{~g} \\
3200 \mathrm{C}\end{array}$ & $\begin{array}{l}1250 \\
3600\end{array}$ \\
\hline $\mathrm{ux}$ de $\mathrm{p}$ & & $2500 \mathrm{c}$ & $3200 \mathrm{c}$ & $3600 \mathrm{c}$ & 4200 \\
\hline
\end{tabular}

Agneaux :

Besoins par roo $\mathrm{g}$ de gain de poids vif.

Animaux àgés d'une semaine
Animaux âgés de deux semaine

Animaux âgés de deux semaines $\ldots \ldots \ldots \ldots$

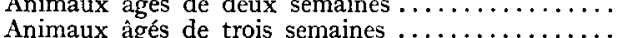

Animaux âgés de quatre semaines..............

$150 \mathrm{~g}$
$180 \mathrm{c}$
$200 \mathrm{c}$
$220 \mathrm{c}$
$225 \mathrm{c}$

$200 \mathrm{~g}$

$200 \mathrm{C}$

$220 \mathrm{C}$

$245 \mathrm{C}$

$260 \mathrm{c}$

$\begin{array}{lll}250 \mathrm{~g} & 350 \mathrm{~g} \\ 220 \mathrm{C} & 250 \mathrm{c} \\ 240 \mathrm{c} & 275 \mathrm{C} \\ 270 \mathrm{C} & 300 \mathrm{C} \\ 300 \mathrm{c} & 350 \mathrm{c}\end{array}$

Porcelets :

Besoins par $100 \mathrm{~g}$ de gain de poids vit.

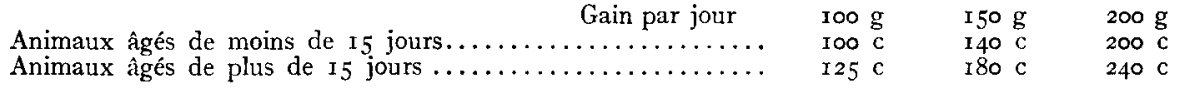

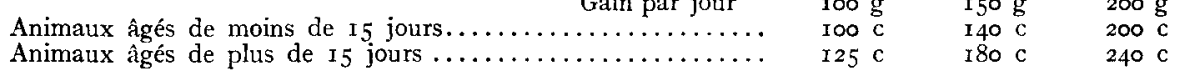

D'après leur composition moyenne, les laits de brebis et de truie ont une valeur calorifique de $\mathrm{I} 200$ calories par $\mathrm{kg}$ pour $8 \%$ de matière grasse avec une teneur en matière sèche correspondante de Igo g, ce qui correspond à I I2o calories d'énergie métabolisable.

Quant au lait de vache, pour une teneur moyenne de $4 \%$ de matière grasse, sa valeur calorifique par $\mathrm{kg}$ est de $75^{\circ}$ calories, avec I30 $\mathrm{g}$ de matière sèche, et 700 calories d'énergie métabolisable.

Pour un animal donné, dont le besoin d'énergie nette est de $e+p$, l'équation à résoudre faisant connaître la quantité de lait nécessaire prend la forme suivante :

I $^{\mathrm{o}}$ Energie métabolisable provenant du lait $=e+p+m s \times \mathrm{I}$ cal, dans laquelle $m s$ correspond à la masse de matière sèche du lait ingéré. 
Désignons maintenant par X la quantité de lait cherchée, et supposons que 1'animal qu'il s'agit de nourrir est un agneau ou un porcelet. Dans ce cas, l'équation I) devient :

$$
\text { I I20 } \times \mathrm{X}=e+p+190 \mathrm{X} \text {, }
$$

équation dans laquelle $\mathrm{X}$ est exprimé en $\mathrm{kg}$.

La résolution de l'équation donne :

$$
\mathrm{X}=\frac{e+p}{930} .
$$

On trouverait de même, en appliquant cette formule au cas du lait de vache, pour la valeur correspondante de $\mathrm{X}$ :

$$
\mathrm{X}=\frac{e+p}{57^{\circ}}
$$

Voici, par exemple, comment il faut calculer la ration de lait nécessaire à un agneau de 2 semaines, qui pèse $8 \mathrm{~kg}$ et gagne $25^{\circ} \mathrm{g}$ par jour :

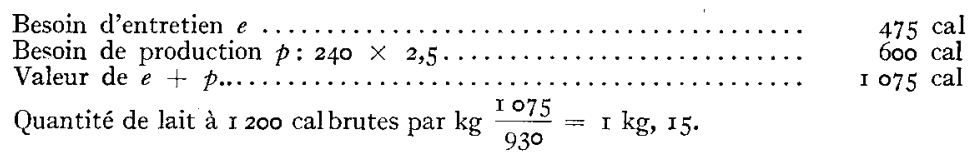

Pour un veau de $80 \mathrm{~kg}$ âgé de 40 jours, qui gagne $\mathrm{I}$ kg de poids vif par jour, le besoin de lait à $4 \%$ de matière grasse se calcule ainsi :

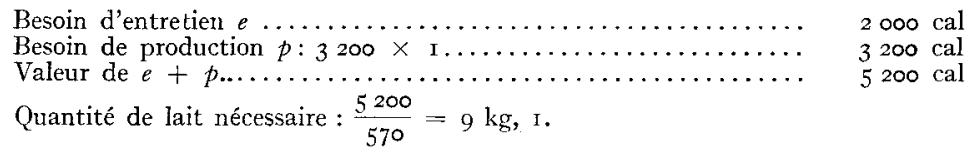

L'un des principaux avantages de cette méthode est de permettre d'évaluer, en quantité de lait ayant la composition moyenne conventionnelle, la production journalière d'une femelle qui allaite sa progéniture. Voici, en effet, de quelle manière il convient d'opérer pour connaître la production laitière d'une truie dont les 8 porcelets, âgés de trois semaines, pèsent en moyenne chacun $5 \mathrm{~kg}$, et gagnent $\mathrm{r} 8 \mathrm{o} \mathrm{g}$ par jour :

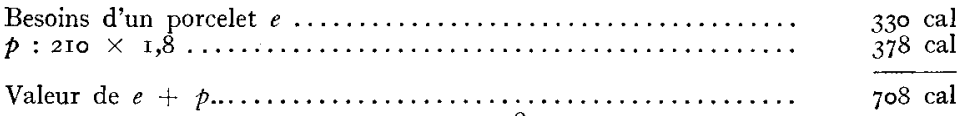

Quantité de lait à $\mathrm{x} 200 \mathrm{cal}$ correspond. $\frac{708}{930}=0 \mathrm{~kg}, 760$

Production de la truie, pour la portée entière : $6 \mathrm{~kg}$, $\mathrm{r}$.

Ein nous servant des données précédentes, nous avons établi les graphiques de la figure 2, qui fournissent par simple lecture la production laitière d'une 
brebis, en fonction de l'âge et du gain journalier de poids vif de son agneau. Ces graphiques montrent que, pour les bonnes laitières, la production, exprimée

\section{Progression des besoins de lagneaw exprimés en quantilés de lait a 1200 cal brutes et $180 \mathrm{gr}$. de mat sèche par $\mathrm{Kg}$.}

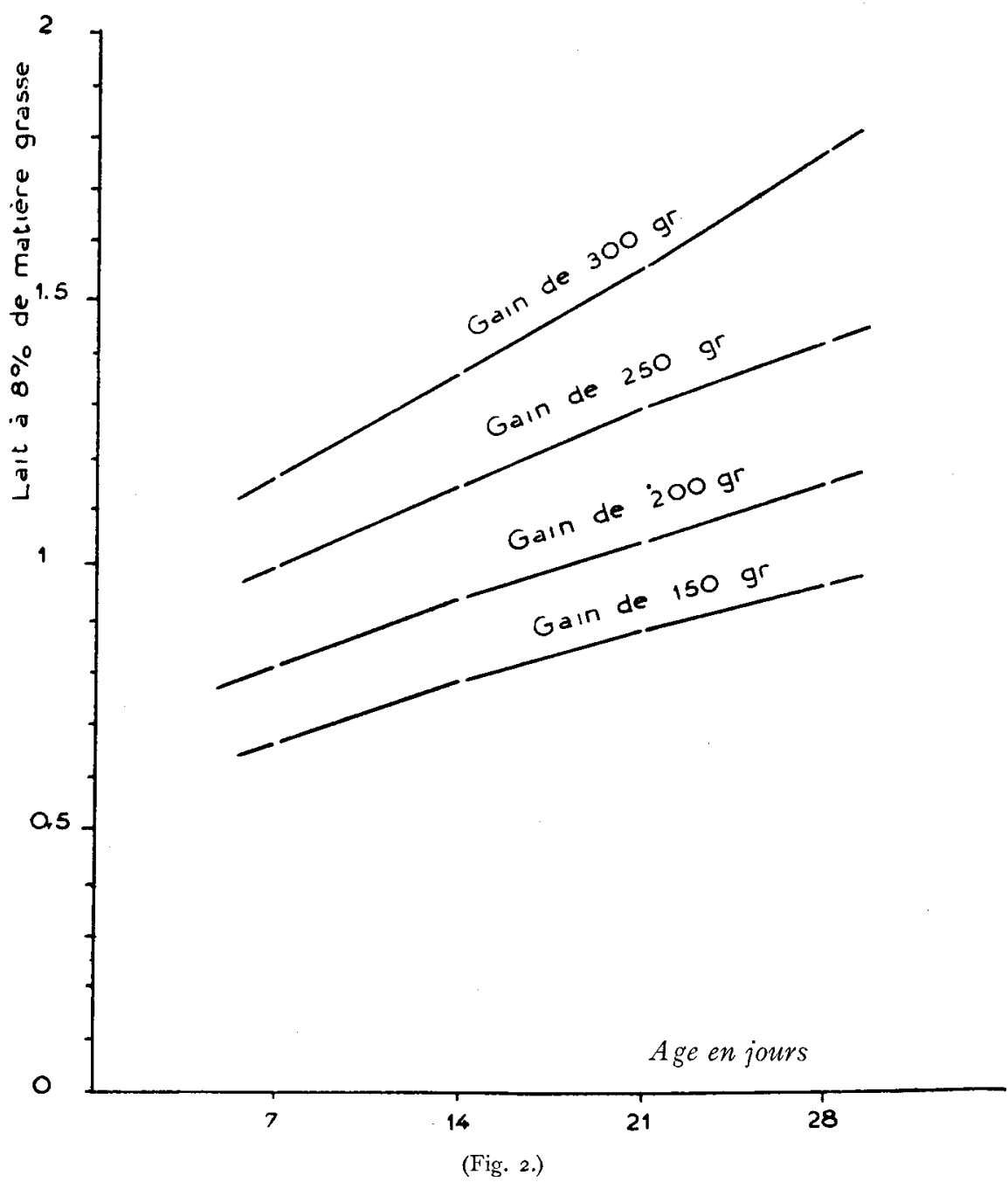

en lait à $8 \%$ de matière grasse, s'élève régulièrement jusqu'à la fin du premier mois, c'est-à-dire à l'âge où l'agneau va commencer à consommer la nourriture mise à sa disposition pour compléter le lait maternel. 
DEUXIEME PARTIE :

\section{UTILISATIONS DE L'AZOTE DU LAIT}

Au cours des I8 expériences précitées de métabolisme effectuées sur des veaux, des agneaux et des porcelets, nous avons mesuré avec exactitude, pour chaque essai, les quantités d'azote ingérées par nos sujets, en même temps que les quantités correspondantes d'azote fécal et d'azote urinaire. Il a donc été possible, dans chaque cas, de calculer le coefficient de rétention de l'azote alimentaire, au moyen de la formule : $\frac{N \text { ingéré }-(N \text { fécal }+N \text { urinaire })}{N \text { ingéré }}$

D'autre part, en tenant compte à la fois des pertes d'azote d'origine endogène, provenant $d u$ tube digestif et des reins, nous avons également tenté d'évaluer dans chaque cas la valeur biologique de l'azote ingéré, en nous servant de la formule classique :

$\mathrm{N}$ ingéré $-[(\mathrm{N}$ fécal total $-\mathrm{N}$ fécal métabolique $)+(\mathrm{N}$ urinaire total $-\mathrm{N}$ urinaire métabolique $)]$ $\mathrm{N}$ ingéré $-(\mathrm{N}$ fécal total $-\mathrm{N}$ fécal métabolique)

Pour l'application de cette formule, nous avons admis que la perte d'azote fécal métabolique était dans tous les cas de $0,64 \mathrm{~g}$ par kilogramme de matière sèche ingérée. En ce qui concerne l'évaluation de l'azote urinaire d'origine endogène, nous avons admis que cette dépense était proportionnelle à la dépense calorifique quotidienne des animaux supposés placés dans les conditions de la mesure du métabolisme basal. Les coefficients utilisés pour ces calculs figurent au tableau ci-après :

TABLEAU VI

Coefficients pour le calcul de la dépense azotée d'origine endogène, en grammes par 1000 calories du métabolisme basal

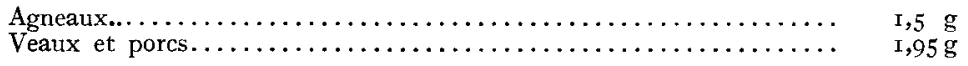

La connaissance du poids des animaux, complétée dans quelques cas par la mesure directe du métabolisme basal sur des sujets à jeun et au tepos nous a permis d'effectuer les calculs indiqués ci-dessus. Nous avons ainsi obtenu les résultats expérimentaux du tableau VII.

A première vue, la variabilité et la faible valeur moyenne des indices ainsi calculés est surprenante, si l'on se souvient que la valeur biologique des protéines du lait, mesurée sur des animaux de laboratoire parvenus à l'âge adulte, est de 85. Mais l'étude attentive des échanges énergétiques de nos sujets 


\section{TABIEAU VII}

Valeurs expérimentales du coefficient de rétention et de la valeur biologique de l'azote ingéré correspondant à chaque cas

\begin{tabular}{|c|c|c|c|c|c|c|c|c|}
\hline \multirow{4}{*}{$\begin{array}{l}\text { No de } \\
\text { l'essai. } \\
-\end{array}$} & \multirow{4}{*}{$\begin{array}{l}\text { Poids du } \\
\text { sujet. } \\
\overline{\mathrm{kg}}\end{array}$} & \multirow{4}{*}{$\frac{N \text { ingéré. }}{\mathrm{g}}$} & \multicolumn{2}{|r|}{$\mathrm{N}$ féral. } & \multicolumn{2}{|r|}{$\mathrm{N}$ urinaire } & \multirow[b]{2}{*}{$\begin{array}{c}\text { C. de } \\
\text { rétention. }\end{array}$} & \multirow[b]{2}{*}{$\begin{array}{l}\text { V. bio- } \\
\text { logique }\end{array}$} \\
\hline & & & total. & métabolique. & total. $\mathrm{m}$ & nétabolique. & & \\
\hline & & & $\bar{g}$ & $\bar{a}$ & $\bar{g}$ & -1 & $\overline{\%}$ & $\%$ \\
\hline & & & & & $\mathrm{g}$ & & $\%$ & $\%$ \\
\hline \multicolumn{9}{|c|}{ Agneaux. } \\
\hline I $\ldots$ & 8,9 & 7,71 & $0,3 \mathrm{I}$ & $0, \mathrm{I} \mathbf{~}$ & 3,38 & 0,75 & 52 & 65 \\
\hline $2 \ldots \ldots \ldots \ldots$ & 9,7 & 6,38 & 0,20 & 0,10 & $3, \pi 5$ & 0,75 & 49 & 6 \\
\hline $3 \ldots$ & II, 2 & 9,36 & 0,35 & 0,16 & 4,04 & 0,82 & 55 & 65 \\
\hline $4 \ldots$ & 7,4 & 6,36 & 0,14 & 0,09 & 2,47 & 0,66 & 60 & 7 \\
\hline $5 \quad \ldots$ & 8,6 & 6,27 & 0,14 & 0,09 & 2,53 & 0,75 & 59 & 71 \\
\hline $6 \quad \ldots$ & 8,2 & 5,50 & 0,28 & 0,09 & 2,02 & 0,70 & $6 \mathrm{I}$ & 75 \\
\hline $7 \ldots$ & 5,2 & 7,59 & 0,30 & $0, \mathrm{II}$ & I,83 & $0,5^{2}$ & 75 & 8 \\
\hline $8 \ldots$ & 5,8 & 6,10 & 0,30 & 0,09 & ז, 99 & 0,60 & 66 & 77 \\
\hline $9 \ldots \ldots \ldots$ & 6,6 & 5,90 & 0,30 & 0,09 & 2,30 & 0,64 & 59 & 71 \\
\hline ro $\ldots \ldots \ldots$. & 6,0 & 5,40 & 0,17 & 0,08 & 1,88 & 0,60 & 64 & 8 \\
\hline I $\ldots \ldots \ldots \ldots$ & 6,7 & 6,57 & 0,17 & 0,10 & 1,85 & 0,64 & $7 \mathrm{I}$ & 8 \\
\hline $12 \ldots \ldots \ldots$ & 7,4 & 4,69 & 0,28 & 0,06 & 2,50 & 0,66 & 4.3 & 5 \\
\hline${ }^{1} 3 \ldots \ldots \ldots$ & 10,3 & II,O3 & 0,35 & 0,15 & 2,86 & 0,79 & 73 & 8 \\
\hline \multicolumn{9}{|c|}{ Veaux } \\
\hline $14 \ldots \ldots \ldots$ & 43,0 & 40,12 & 3,32 & 0,60 & 13,30 & 3,36 & 64 & 73 \\
\hline$I_{5} \ldots \ldots \ldots \ldots$ & 44,0 & 28,24 & 2,89 & 0,43 & 10,35 & 3,33 & 59 & \\
\hline I6 $\ldots \ldots \ldots \ldots$ & 48,6 & $72, \mathrm{I} 2$ & 22,52 & 0,77 & 22,34 & 3,72 & 55 & 6 \\
\hline I $7 \ldots \ldots \ldots$ & 53,0 & 53,16 & $\begin{array}{r}\mathrm{r}, 99 \\
\end{array}$ & 0,80 & $\begin{array}{l}19,37 \\
\end{array}$ & 3,84 & 62 & \\
\hline \multicolumn{9}{|c|}{ Porcelets } \\
\hline $18 \ldots \ldots \ldots$ & & 13,9 & 0,6 & 0,2 & 4,2 & 0,7 & 68 & 74 \\
\hline
\end{tabular}

nous montre que les jeunes animaux exclusivement nourris de lait utilisent pour leur régulation thermique, ainsi que pour le fonctionnement de leurs organisme, c'est à dire et pour les réactions qui sont la condition de la vie cellulaire, une fraction importante de l'énergie métabolisable provenant des matières azotées ingérées. Les données recueillies par nos soins nous ont permis dans chaque cas de mesurer la valeur de cette fraction. Pour ne pas compliquer notre exposé, nous nous sommes bornés à reprođuire en détail les résultats de deux expériences (expérience $n^{0} I$, sur agneau, et expérience $n^{0} r_{4}$, sur veau).

\section{TABLEAU VIII}

\section{Bilans d'utilisation de l'Energie Métabolisable}

Expérience $\mathrm{N}^{0}$ I sur agneau

Sources de l'énergie métabolisable: Utilisation de l'énergie métabolisable :

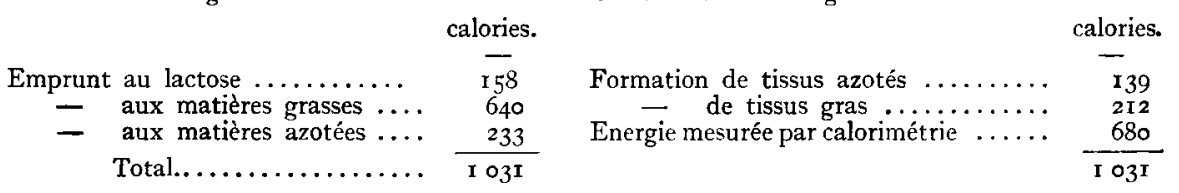


Particularités de l'utilisation de l'énergie métabolisable des matières azotées :

calories.

Energie retenue sous forme de tissus de croissance............

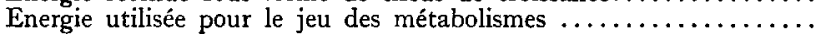

Total de l'énergie métabolisable provenant des matières azotées ...

Fraction de l'énergie des matières azotées utilisée pour le jeu des métabolismes

$$
\begin{array}{r}
\overline{139} \\
94 \\
\hline 233
\end{array}
$$

Expérience $\mathrm{N}^{0}$ I4 sur veau

Sources de l'énergie métabolisable:

Utilisation de l'énergie métabolisable

calories.

calories.

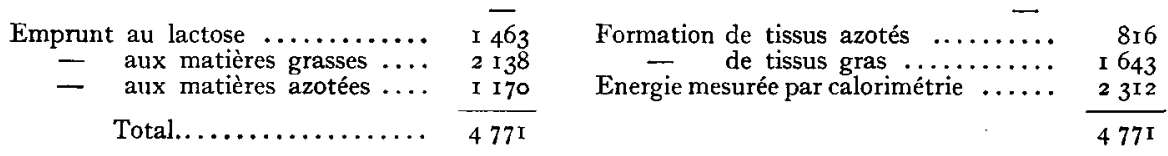

Particularités de l'utilisation de l'énergie métabolisable des matières azotées :

Energie retenue sous forme de tissus de croissance............

Energie utilisée pour le jeu des métabolismes $\ldots \ldots \ldots \ldots \ldots \ldots \ldots$

Total de l'énergie métabolisable provenant des matières azotées ...

Fraction de l'énergie des matières azotées utilisée pour le jeu des méta-

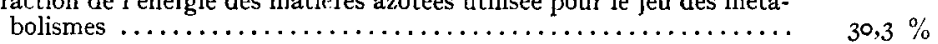

Le tableau IX ci-joint montre quelle a été, pour chacune des I8 expériences, la répartition de l'énergie métabolisable provenant des matières azotées entre l'énergie mise en réserve sous forme de tissus de croissance, d'une part, et l'énergie dissipée à l'extérieur à la suite du jeu des métabolismes, d'autre part.

\section{TABLEAU IX}

Répartition de l'énergie métabolisable provenant des matières azotées

No de

l'essai.

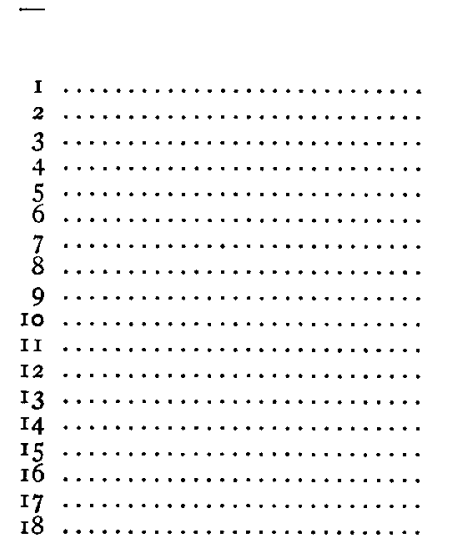

Energie
métabolisable
provenant
des matières
azotées.

$\overline{(a)}$
calories.
233
193
294
203
191
167
242
186
180
167
205
132
356
I 170
777
I 626
I 646
447

Energie
retenue
sous forme
de tissus de
croissance.

Energie dissipée par le jeu Rapport des méta-
bolismes.

calories.

$$
\begin{array}{r}
\overline{816} \\
354 \\
\hline
\end{array}
$$

$\begin{array}{rl}\begin{array}{c}(c) \\ \text { alories. }\end{array} & - \\ 94 & \\ 88 & 40,4 \\ \mathrm{r22} & 45,5 \\ 73 & 4 \mathrm{I}, 5 \\ 66 & 36,0 \\ 56 & 34,6 \\ 53 & 33,5 \\ 54 & 2 \mathrm{I}, 7 \\ 66 & 29, \mathrm{I} \\ 5 \mathrm{I} & 36,7 \\ 47 & 30,7 \\ 70 & 22,9 \\ 85 & 53,0 \\ 354 & 23,9 \\ 257 & 30,3 \\ 679 & 33, \mathrm{I} \\ 543 & 4 \mathrm{I}, 7 \\ \mathrm{r} 39 & 33,0 \\ & 3 \mathrm{I}, \mathrm{I}\end{array}$


En nous servant des données des tableaux précédents, nous avons construit les graphiques de la fig. 3, en portant en abscisses les rapports de la dernière

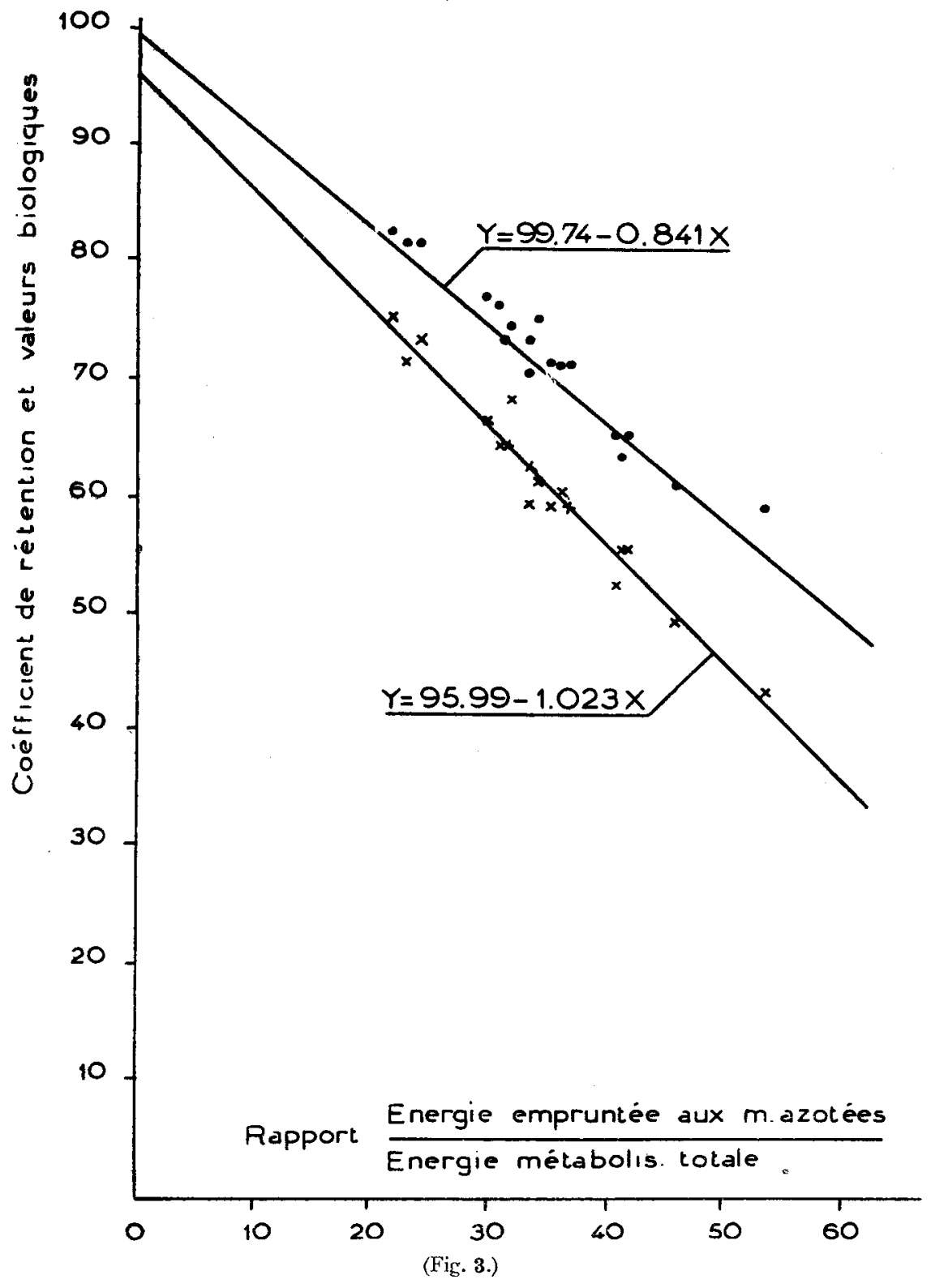

colonne du tableau IX, d'une part, et d'autre part, les valeurs correspondantes des coefficients de rétention (représentées par des croix) et des valeurs biologiques (représentées par des points).

Il suffit d'un simple examen de ces graphiques pour se rendre compte que 
les deux séries de points et de croix sont alignées sur des droites dont il est aisé de calculer les paramètres par la méthode des moindres carrés. Nous trouvons ainsi pour la première de ces droites, qui se rapporte aux valeurs biologiques, l'équation :

I)

$$
y=99,74-0.841 x
$$

et pour la seconde, qui correspond aux coefficients de rétention .

2)

$$
y=95.99-1,023^{x}
$$

Nous en concluons que dans l'hypothèse où les besoins énergétiques de l'animal pourraient être satisfaits en totalité par des glucides et des lipides, la transformation de la matière azotée du lait en matière azotée des tissus de croissance pourrait se faire avec un coefficient de perte insignifiant, ce qui revient à dire que la valeur biologique mesurée dans ces conditions devrait être très voisine de roo. De son côté, le coefficient de rétention, qui se rapproche de la valeur biologique à mesure que l'énergie empruntée aux matières azotées pour 1e jeu des métabolismes diminue, prend une valeur limite de 96 , dans 1'hypothèse de la suppression de cet emprunt.

\section{CONCLUSIONS}

De l'ensemble de ces travaux, il est possible de déduire les conclusions suivantes :

$I^{\circ}$ La connaissance du mécanisme de 1'utilisation de l'énergie contenue dans les matériaux ingérés avec le lait permet de calculer avec une approximation suffisante les quantités de lait nécessaires à de jeunes animaux d'un poids donné dont on veut obtenir un gain quotidien déterminé. L'emploi de la méthode ainsi définie permet également de connaître la production laitière d'une femelle allaitant ses petits, en fonction de la vitesse de croissance de ces derniers.

$2^{\circ}$ L'utilisation de l'azote du lait est limitée par la nécessité dans laquelle se trouve l'organisme de faire appel aux protides de sa ration pour couvrir partiellement ses besoins énergétiques. Le coefficient de rétention et la valeur biologique de cet azote dépendent du niveau de nutrition de l'animal et sont d'autant plus éloignés de leurs valeurs limites que le sujet est moins bien alimenté. 


\section{Tableau A}

Ingesta, Excreta, Balance de l'azote

Fèces Urine

Azote

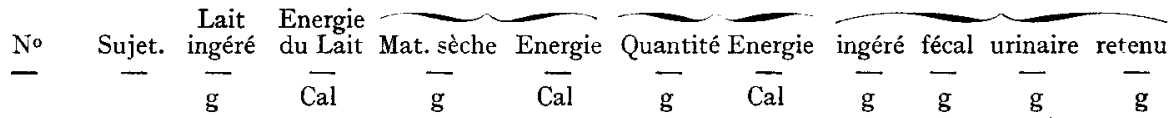

Expériences sur agneaux

\begin{tabular}{|c|c|c|c|c|c|c|c|c|c|c|c|}
\hline$[\quad \cdots$ & D & $85 I$ & $I I I 7$ & 9,63 & 56 & 403 & 30 & $7,7 \mathrm{I}$ & 0,31 & $3,3^{8}$ & 4,02 \\
\hline $2 \ldots$ & $\mathrm{G}$ & 137 & 993 & 5,42 & 20 & 347 & 27 & $6,3^{8}$ & 0,20 & 3,15 & 3,03 \\
\hline .. & O & I 137 & I 729 & $4, \mathrm{I} 2$ & r9 & 434 & 28 & $9,3^{6}$ & 0,35 & 4,04 & 4,97 \\
\hline .. & $28 \mathrm{M}$ & $8 \mathrm{I} 5$ & 9 I 5 & 3,01 & $r_{3}$ & 606 & $2 \mathrm{I}$ & $6,3^{6}$ & 0,14 & 2,47 & 3,75 \\
\hline . & $28 \mathrm{M}$ & $73^{6}$ & 836 & 3,01 & I3 & 393 & 27 & 6,27 & $0, \mathrm{I} 4$ & 2,53 & 3,60 \\
\hline$\ldots$ & $28 \mathrm{~F}$ & 710 & 900 & 6,10 & 30 & 297 & 20 & $7,5^{\circ}$ & 0,28 & 2,02 & 3,20 \\
\hline .. & $28 \mathrm{M}$ & 875 & I 075 & 3,89 & I 7 & 567 & I9 & 5,59 & 0,30 & 1,83 & 5,46 \\
\hline . & $28 \mathrm{M}$ & 784 & 942 & 3,89 & I 7 & 399 & $2 \mathrm{I}$ & 6,10 & 0,30 & 1,99 & 3,81 \\
\hline . & $28 \mathrm{M}$ & 775 & 947 & 3,89 & I 7 & 399 & 21 & 5,90 & 0,30 & 2,30 & 3,30 \\
\hline . . & $28 \mathrm{~F}$ & 705 & 766 & $2,5^{8}$ & I3 & $42 I$ & 19 & $5,4^{\circ}$ & 0,17 & $\mathrm{I}, 88$ & 3,35 \\
\hline . & $28 \mathrm{~F}$ & 835 & 970 & $2,5^{8}$ & I3 & 433 & 21 & 6,57 & $0, \mathrm{I} 7$ & $\mathrm{r}, 85$ & 4,55 \\
\hline . & $28 \mathrm{~F}$ & $6 r o$ & 630 & 6,10 & 30 & 528 & 25 & 4,69 & 0,28 & 2,50 & $\mathrm{I}, 9 \mathrm{I}$ \\
\hline . & $28 \mathrm{~F}$ & I 327 & I $\mathbf{3 2 8}$ & $10,6 \mathrm{I}$ & 53 & 772 & 24 & II, 03 & 0,35 & 2,86 & 7,82 \\
\hline
\end{tabular}

Expériences sur veau

\begin{tabular}{|c|c|c|c|c|c|c|c|c|c|c|}
\hline$\ldots \ldots \ldots$ & 7683 & $\begin{array}{l}5356 \\
3866\end{array}$ & - & $44^{2}$ & - & $\begin{array}{l}\text { I } 43 \\
26\end{array}$ & 40, I 2 & $\begin{array}{r}3,32 \\
2,80\end{array}$ & 13,30 & $\begin{array}{l}23,5^{\circ} \\
15,00\end{array}$ \\
\hline Lait écrémé & $\begin{array}{l}5400 \\
8000\end{array}$ & & - & $3^{20}$ & - & & 20,24 & & 10,35 & \\
\hline Lait sec & $\begin{array}{l}500 \\
10000\end{array}$ & $\begin{array}{l}5290 \\
7155\end{array}$ & & $\begin{array}{l}269 \\
242\end{array}$ & - & $\begin{array}{l}145 \\
182\end{array}$ & $\begin{array}{l}72,16 \\
53,16\end{array}$ & $\begin{array}{r}22,52 \\
1,99\end{array}$ & $\begin{array}{l}22,34 \\
19,37\end{array}$ & $\begin{array}{l}27,3^{\circ} \\
31,80\end{array}$ \\
\hline
\end{tabular}

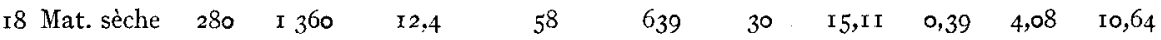

\section{Tableau B}

\section{Bilans du Carbone et de l'Energie}

Carbone

Energie

No Sujet ingéré fécal urinaire $\mathrm{du}_{\mathrm{CO}}$ retenu Aliments Fèces Urine Croît Disparue

\begin{tabular}{|c|c|c|c|c|c|c|c|c|c|c|c|}
\hline 一 & - & $\frac{-}{g}$ & $\overline{\mathrm{g}}$ & $\overline{\mathrm{g}}$ & $\overline{\mathrm{g}}$ & $\overline{\mathrm{g}}$ & $\overline{\mathrm{cal}}$ & $\overline{\mathrm{cal}}$ & $\overline{\mathrm{cal}}$ & $\overline{\mathrm{cal}}$ & cal \\
\hline \multicolumn{12}{|c|}{ Expériences sur agneaux } \\
\hline I $\ldots \ldots$ & $\mathrm{D}$ & 98,2 & 5,3 & 1,7 & 60,2 & $3^{r, o}$ & 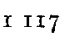 & 56 & 30 & $35^{\mathrm{I}}$ & 710 \\
\hline $2 \ldots \ldots$ & $\mathrm{G}$ & 87,2 & 2,4 & I, 6 & 60,5 & 22,7 & 993 & 20 & 27 & 257 & 689 \\
\hline $3 \ldots \ldots$ & $\mathrm{O}$ & I 50,4 & $\mathbf{I}, 9$ & 2,0 & 73,5 & $73, \circ$ & I 729 & I9 & 28 & 847 & 835 \\
\hline $4 \ldots \ldots$ & $28 \mathrm{M}$ & 80,9 & $\mathrm{I}, 4$ & 1,2 & 55,0 & $23: 3$ & 9 r 5 & I3 & $2 \mathrm{I}$ & 262 & 619 \\
\hline $5 \ldots \ldots \ldots$ & $28 \mathrm{M}$ & 74,4 & $I, 4$ & I,3 & $5^{6,5}$ & I 5,2 & 836 & I3 & 27 & 162 & 634 \\
\hline $6 \ldots \ldots$ & $28 \mathrm{~F}$ & 78,9 & 2,7 & 1,0 & 54,2 & $2 \mathrm{I}, \mathrm{O}$ & 900 & 30 & 20 & 235 & 615 \\
\hline $7 \ldots \ldots$ & $28 \mathrm{M}$ & 94,9 & $x, 7$ & 0,9 & 47,9 & 44,4 & 1075 & I 7 & r9 & 504 & 535 \\
\hline $8 \ldots \ldots$ & $28 \mathrm{M}$ & $83, \mathrm{I}$ & $\mathrm{I}, 7$ & 1,0 & 49,2 & $3^{\mathrm{I}, 2}$ & 942 & I 7 & $2 \mathrm{I}$ & 354 & 554 \\
\hline $9 \ldots \ldots$ & $28 \mathrm{M}$ & 83,3 & $\mathrm{I}, 7$ & $\mathrm{I}, 7$ & 50,5 & 29,4 & 947 & I7 & $2 \mathrm{I}$ & 335 & 574 \\
\hline $10 \ldots \ldots \ldots$ & $28 \mathrm{~F}$ & $68, \mathrm{I}$ & $\mathrm{I}, 2$ & 0,9 & 50,8 & $x_{5,2}$ & 766 & I3 & I9 & 164 & 570 \\
\hline$y+\ldots \ldots$ & $28 \mathrm{~F}$ & 85,8 & 1,2 & 0,9 & $50, \mathrm{r}$ & 33,6 & 970 & I3 & $2 \mathrm{I}$ & 382 & 554 \\
\hline $12 \ldots \ldots$ & $28 \mathrm{~F}$ & 55,9 & 2,7 & $\mathrm{I}, 2$ & $5^{2,4}$ & $-0,2$ & 630 & 30 & 25 & -7 & $5^{8} 3$ \\
\hline $13 \ldots \ldots$ & $28 \mathrm{~F}$ & I I 8,9 & 4,8 & $\mathrm{I}, 4$ & 65,3 & 47,4 & I 328 & 53 & 24 & $5^{28}$ & 723 \\
\hline \multicolumn{12}{|c|}{ Expériences sur veau } \\
\hline$I_{4} \ldots \ldots \ldots$ & $v$ & 485 & 32 & I 5 & 221 & 217 & $535^{6}$ & 442 & I43 & 2459 & 23 I 2 \\
\hline $1_{5} \ldots \ldots$ & $v$ & 349 & 22 & I2 & 2 I 5 & 100 & 3866 & 320 & I 26 & I I 24 & 2288 \\
\hline I6 $\ldots$ & $v$ & 516 & 9 & 38 & 283 & I 86 & 5296 & 269 & I 43 & 2095 & 2787 \\
\hline I $7 \ldots \ldots \ldots$ & $v$ & 647 & I 7 & 27 & 283 & 320 & 7155 & 242 & I82 & 3678 & 3053 \\
\hline
\end{tabular}

Expériences sur porcelets

I8

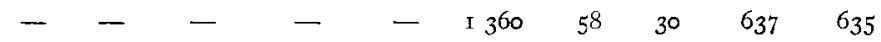


No

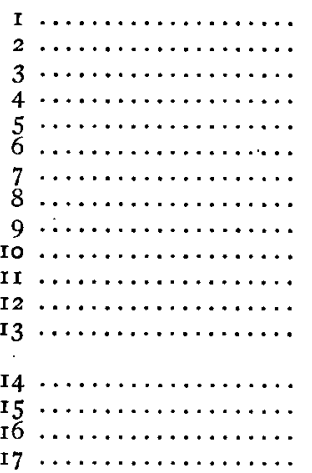

I8
TABLEÁU C

\section{Analyse des Eléments du croît}

Matières azotées du croît Matières grasses du croît Sujet Gain journalier Energie Gain journalier Energie $\overline{\mathrm{g}} \quad \overline{\mathrm{cal}}$

Expériences sur agneaux

\begin{tabular}{|c|c|c|c|c|}
\hline D & $25, \mathrm{I}$ & I 39 & 23,0 & 212 \\
\hline $\mathrm{G}$ & $\mathbf{1 8 , 9}$ & ro5 & 16,5 & I $5^{2}$ \\
\hline $\mathrm{O}$ & $3 I, I$ & I 73 & 73,3 & 674 \\
\hline $28 \mathrm{M}$ & 23,4 & I 30 & $\mathrm{I} 4,3$ & I 32 \\
\hline $28 \mathrm{M}$ & 22,5 & I 25 & 4,0 & 37 \\
\hline $28 \mathrm{~F}$ & 20,0 & I I I & I3, I & I 2 I \\
\hline $28 \mathrm{M}$ & $34, \mathrm{I}$ & 189 & 34,2 & $3 \mathbf{I} 5$ \\
\hline $28 \mathrm{M}$ & 23,8 & $I^{2}$ & $24, I$ & 222 \\
\hline $28 \mathrm{M}$ & 20,6 & I I 4 & 24,0 & 221 \\
\hline $28 \mathbf{F}$ & 20,9 & I 16 & 5,2 & 48 \\
\hline $28 \mathrm{~F}$ & 28,4 & I 58 & 24,3 & 224 \\
\hline $28 \mathrm{~F}$ & $I I, 9$ & 66 & $-7,5$ & -69 \\
\hline $28 \mathrm{~F}$ & 48,9 & $27 \mathrm{I}$ & 28,0 & 257 \\
\hline \multicolumn{5}{|c|}{ Expériences sur veau } \\
\hline$v$ & I 47 & 815 & 178 & I 643 \\
\hline$v$ & 94 & 520 & 66 & 604 \\
\hline$v$ & I $7 \mathrm{I}$ & I I 22 & 106 & 973 \\
\hline$v$ & I99 & I IO3 & 279 & 2575 \\
\hline
\end{tabular}

Expériences sur porcelets

$66,5 \quad 369$

29,2

268 